\title{
Comparison between robotic and laparoscopic inguinal hernia repair in Caucasian patients: a systematic review and meta- analysis
}

\author{
Fenglin Zhao, Baoshan Wang, Jie Chen \\ Department of Hernia and Abdominal Wall Surgery, Beijing Chao-Yang Hospital, Beijing, China \\ Contributions: (I) Conception and design: F Zhao, J Chen; (II) Administrative support: F Zhao, B Wang; (III) Provision of study materials or patients: \\ F Zhao, B Wang; (IV) Collection and assembly of data: F Zhao, B Wang; (V) Data analysis and interpretation: F Zhao, J Chen; (VI) Manuscript \\ writing: All authors; (VII) Final approval of manuscript: All authors. \\ Correspondence to: Jie Chen, MD. Department of Hernia and Abdominal Wall Surgery, Beijing Chao-Yang Hospital, No.5 Jingyuan Road, Shijingshan \\ District, Beijing 100043, China. Email: chenjiejoe@sina.com.
}

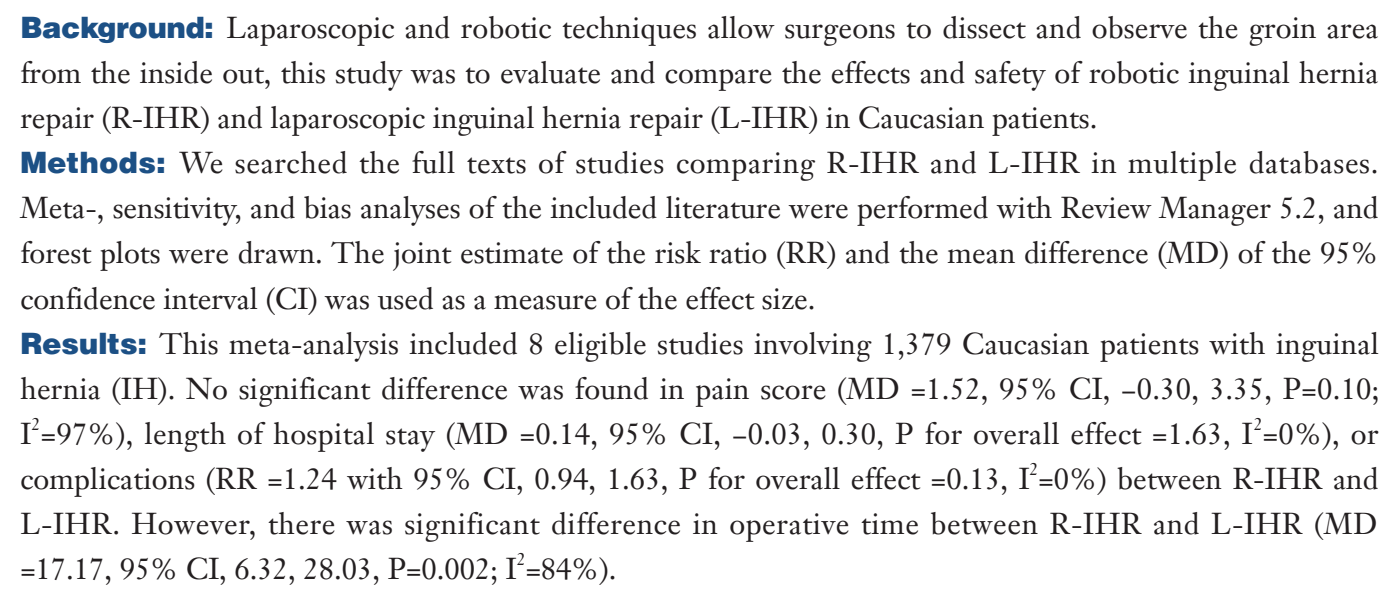

Discussion: This meta-analysis revealed only minor differences between R-IHR and L-IHR in terms of clinical effects and safety in Caucasian patients, although R-IHR has a longer operative time than L-IHR. Both R-IHR and L-IHR are suitable to treat Caucasian patients with IH.

Keywords: Robotic inguinal hernia repair (R-IHR); laparoscopic inguinal hernia repair (L-IHR); Caucasian; meta-analysis

Submitted Apr 06, 2021. Accepted for publication May 19, 2021.

doi: 10.21037/atm-21-2126

View this article at: http://dx.doi.org/10.21037/atm-21-2126

\section{Introduction}

The inguinal area is located in the triangular area between the lower abdominal wall and the thigh. Inguinal hernia (IH), commonly known as "hernia", refers to a mass formed when the abdominal visceral organs protrude through the body's surface due to a defect in the inguinal area $(1,2)$. In older people, muscle atrophy, a weak abdominal wall, and a weakened inguinal area provides a channel for hernia formation. Furthermore, the development of cough or asthma in older people increases the abdominal pressure and the risk of developing hernia (3). There is a sex difference in the incidence of hernia, with the female and male incidence reaching about $3 \%$ and $27 \%$, respectively. Achieving selfcure of IH is challenging; therefore, IH surgery has become one of the most common abdominal surgical procedures $(4,5)$. With the concept of minimally invasive surgery having 
been accepted by the public in recent years, laparoscopic hernia repair has been widely applied.

Laparoscopic IH repair (L-IHR) is a safe and tension-free procedure. The process of laparoscopic preperitoneal patch repair is to enter the abdominal cavity, and firstly open the peritoneum around the hernia defect, then place the patch on the defect, and finally close the peritoneum. The advantages of L-IHR include small incisions, good aesthetics, fast patient recovery, and high patient satisfaction, however, require high cardiopulmonary function to patients due to general anesthesia operation $(6,7)$.

With the increasing application of the Da Vinci robotic surgical system in abdominal surgery in recent years, robotic assistance has also been incorporated into IH repair (IHR). The robotic arm of the system can perform operations that require high flexibility in an extremely small space $(8,9)$. The application of a robotic surgical system in laparoscopic surgery can increase the surgical precision.

Several studies have shown that although robotic IHR (R-IHR) takes longer to perform, postoperative pain is reduced compared to that with traditional laparoscopy, and the postoperative recovery is faster (8-10). The anatomical structure of the groin region is highly complex, with a large number of blood vessels and nerves. The advantages of robotic surgical system can also make the anatomy clearer through its 3-dimensional (3D) high-definition imaging system, which greatly reduces or even avoids the damage sustained by nerves and blood vessels during surgery.

Several randomized trials have compared R-IHR and L-IHR in Caucasian patients. However, previous reviews of IH lacked a consistent comparator. To address these issues, we conducted a meta-analysis of randomized trials and casecontrol studies to compare the effects and safety of R-IHR and L-IHR in Caucasian patients. We present the following article in accordance with the PRISMA reporting checklist (available at http://dx.doi.org/10.21037/atm-21-2126).

\section{Methods}

\section{Literature search strategy}

We searched electronic databases for articles comparing the effects and safety of R-IHR and L-IHR among Caucasian patients. The search databases included PubMed, EMBASE, Cochrane Library, and China National Knowledge. Searches were conducted to identify studies published from May 2000 to May 2019, using the following keywords: (I) robotic inguinal hernia repair or R-IHR; (II) laparoscopic inguinal hernia repair or L-IHR; and (III) inguinal hernia repair or IH. All keyword combinations were connected with "AND", and no language restrictions were applied. To maximize the number of inquiries, we also checked the retrieved studies' references to find other relevant studies.

\section{Study selection}

First, we conducted primary screening, followed by a review of potentially relevant research texts. To be included, studies needed to meet the following criteria: (I) a comparative study of L-IHR and R-IHR; (II) study participants had IR; (III) study participants were Caucasian; and (IV) the full text was available.

Studies meeting any of the following conditions were excluded: (I) research on other health issues; (II) participants received treatments other than L-IHR or R-IHR; (III) the study participants were not Caucasian; or (IV) lacking available data for research.

\section{Data extraction and quality assessment}

Two authors reviewed the full papers of eligible studies. From each article, they extracted data including the first author's name, publication year, participants' age and sex, country, sample size, years of onset for each article. The methodological quality of the study was assessed using the Cochrane risk-of-bias tool. We assessed the quality of the included studies based on the Review Manager 5.2 riskof-bias summary table. The studies were assessed for the following: (I) random sequence generation, (II) allocation concealment, (III) blinding of participants and personnel, (IV) blinding of outcome, (V) incomplete outcome data, (VI) selective reporting (reporting bias) and (VII) other biases (11).

\section{Statistical analysis}

We used Review Manager 5.2 software (Cochrane Collaboration, 2011) to evaluate the difference in comparative analysis results between R-IHR and L-IHR.

Continuous data were evaluated using the mean difference (MD ). The risk ratio (RR) was used for the evaluation of binary data, and $95 \%$ confidence intervals (CIs) were calculated to determine the clinical efficacy and complications of R-IHR and L-IHR. Heterogeneity was assessed using the inconsistency index $\left(\mathrm{I}^{2}\right)$ statistical method, with the $\mathrm{I}^{2}$ statistical value reflecting the level of heterogeneity. When $\mathrm{I}^{2}$ was greater than $50 \%$, 


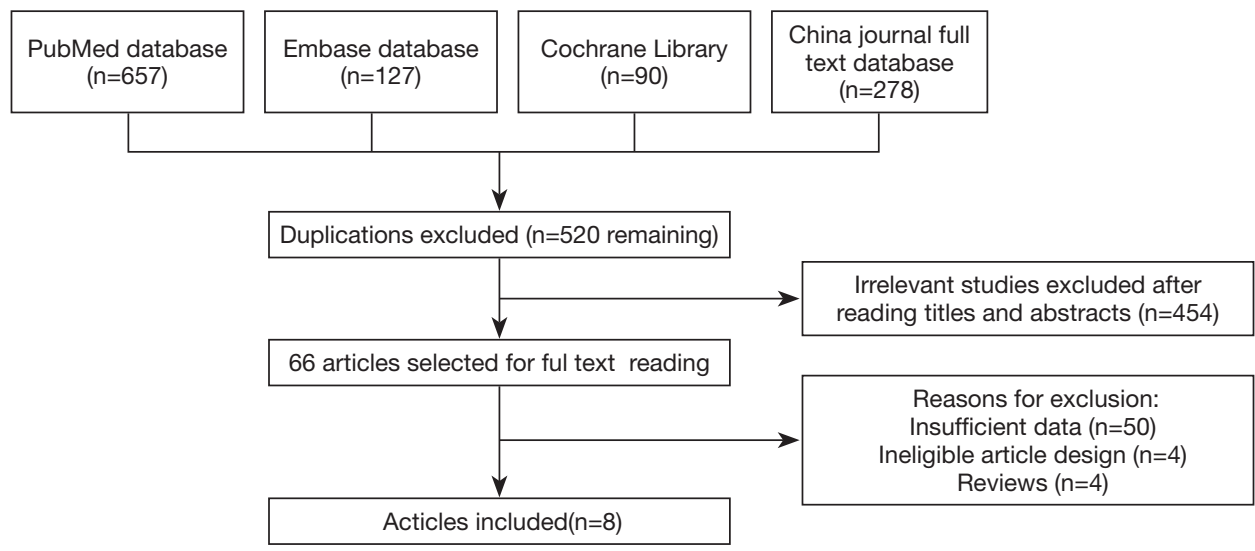

Figure 1 Flow diagram of the study selection process.

heterogeneity was considered to exist, and the randomeffects model was adopted; otherwise, the fixed-effects model was adopted. $\mathrm{P}<0.05$ was considered to be statistically significant. Review Manager 5.2 software was also used to conduct a bias analysis of the studies to test their quality. A funnel chart was used to estimate publication bias.

\section{Surgical procedures}

The L-IHR procedure was performed as follows. In front of the hernia ring defect, the peritoneum was cut in an arc from the anterior superior iliac crest to the medial umbilical ligament, and then the combined Retzius gap and Bogros gap were separated. After the hernia sac had been visualized and checked for any bleeding, a polypropylene fiber patch was inserted through the operation hole forceps and stretched to fully cover the defect area. Finally, multi-point fixation with bio glue was used to continuously suture the local peritoneum closed using absorbable sutures (7).

The Da Vinci robotic transperitoneal hernia surgical procedure (R-IHR) was performed as follows. Using an open method, a pneumoperitoneum was established by placing a $12-\mathrm{mm}$ cannula approximately $2 \mathrm{~cm}$ above the healthy side of the umbilical foramen. The Da Vinci 3D lens was placed with the lens facing up. An 8-mm robot cannula was placed under the camera at the level of the lateral flat umbilicus outside the rectus abdominis and at the level of the lateral margin of the lateral rectus abdominis under the umbilicus. The host was pushed in from the affected foot , and the DaVinci system 3D laparoscope was connected to the observation hole. The $1^{\text {st }}$ arm was connected to the main operation hole, and the $2^{\text {nd }}$ arm was connected to the auxiliary operation hole. Bipolar coagulation and grasping forceps were placed respectively. After gaining entry to the abdomen, the surgeon first to confirmed the type of hernia, and then the peritoneum was cut from the inner umbilical folds to the anterior superior iliac crest on the upper edge of the hernia (8).

\section{Results}

\section{Search process}

After our initial screening, there were 520 articles, among which 66 papers met the preliminary criteria. Of these 66 articles, 58 were excluded due to the study design, having insufficient data, or the article type. Eventually, 8 papers involving 1,120 patients were selected for the metaanalysis. The flowchart in Figure 1 shows the identification, inclusion, and exclusion processes.

\section{Information of included studies}

Table 1 summarizes the study information including the first author, publication year, country, and participant age range. The 8 articles $(7,12-18)$ in this meta-analysis were all published between 2011 and 2019. The sample size of the included studies ranged from 38 to 275 participants. Of the 1,120 patients in the 8 included studies, 459 cases were in the R-IHR group and 661 cases were in the L-IHR group.

\section{Results of quality assessment}

The Cochrane bias risk tool was used to assess the possible bias of the included 8 studies (Figure 2 and Figure S1). 
Table 1 Characteristics of studies included in the meta-analysis

\begin{tabular}{|c|c|c|c|c|c|c|c|}
\hline Study & Year & Language & Country & Age range (mean) & Groups & $\mathrm{n}$ & Years of onset \\
\hline Bittner et al. (12) & & & & & L-IHR & 83 & \\
\hline \multirow[t]{2}{*}{ Huerta et al. (13) } & 2019 & English & USA & $59.1 \pm 12.5$ & R-IHR & 71 & 2005-2017 \\
\hline & & & & & L-IHR & 128 & \\
\hline Khoraki et al. (14) & & & & & L-IHR & 138 & \\
\hline \multirow[t]{2}{*}{ Kudsi et al. (15) } & 2017 & English & USA & $56.9 \pm 15.1$ & R-IHR & 118 & 2012-2015 \\
\hline & & & & & L-IHR & 157 & \\
\hline Muysoms et al. (16) & 2018 & English & USA & $59.7 \pm 14.2$ & R-IHR & 49 & 2013-2017 \\
\hline Sheldon et al. (17) & & & & & L-IHR & 34 & \\
\hline \multirow[t]{2}{*}{ Spernat et al. (7) } & 2014 & English & USA & $59.2 \pm 10.2$ & R-IHR & 5 & 2004-2010 \\
\hline & & & & & L-IHR & 33 & \\
\hline \multirow[t]{2}{*}{ Waite et al. (18) } & 2016 & English & USA & $57.8 \pm 11.5$ & R-IHR & 39 & 2012-2014 \\
\hline & & & & & L-IHR & 24 & \\
\hline
\end{tabular}

R-IHR, robotic inguinal hernia repair; L-IHR, laparoscopic inguinal hernia repair.

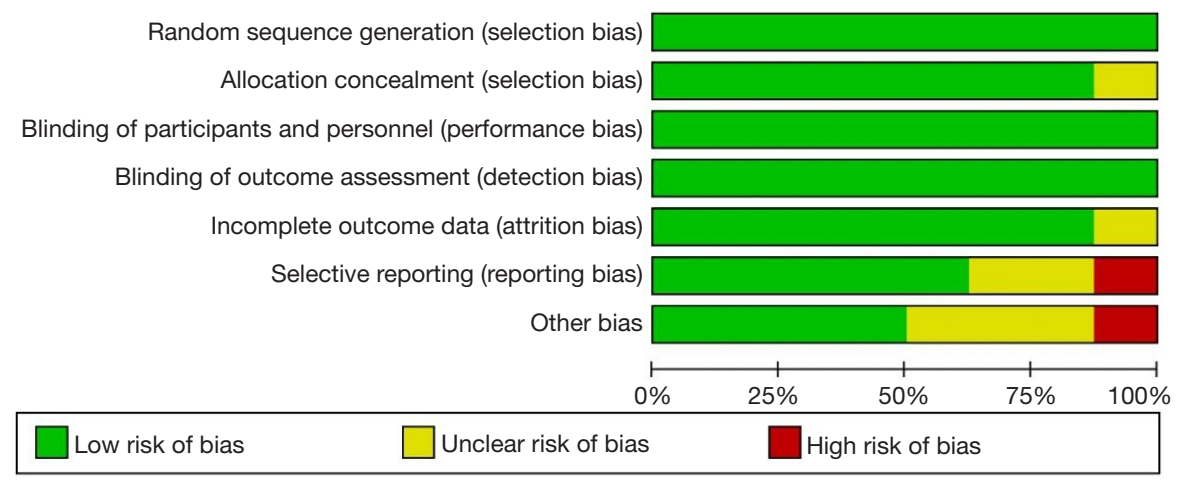

Figure 2 Assessment of the quality of the included studies. Green represents as low risk of bias, yellow represents as unclear risk of bias, and red represents as high risk of bias.

The results of the bias risk analysis revealed that 1 study had reporting bias and 1 study had other biases. Overall, no issues were found with selection bias, attrition bias, performance bias, or detection bias. Therefore, 2 studies had a risk of bias and 6 studies had no risk of bias.

\section{Results of heterogeneity test}

Comparison of the pain score between R-IHR and L-IHR

Four of the included studies compared pain scores between Caucasian patients treated with R-IHR and L-IHR. 


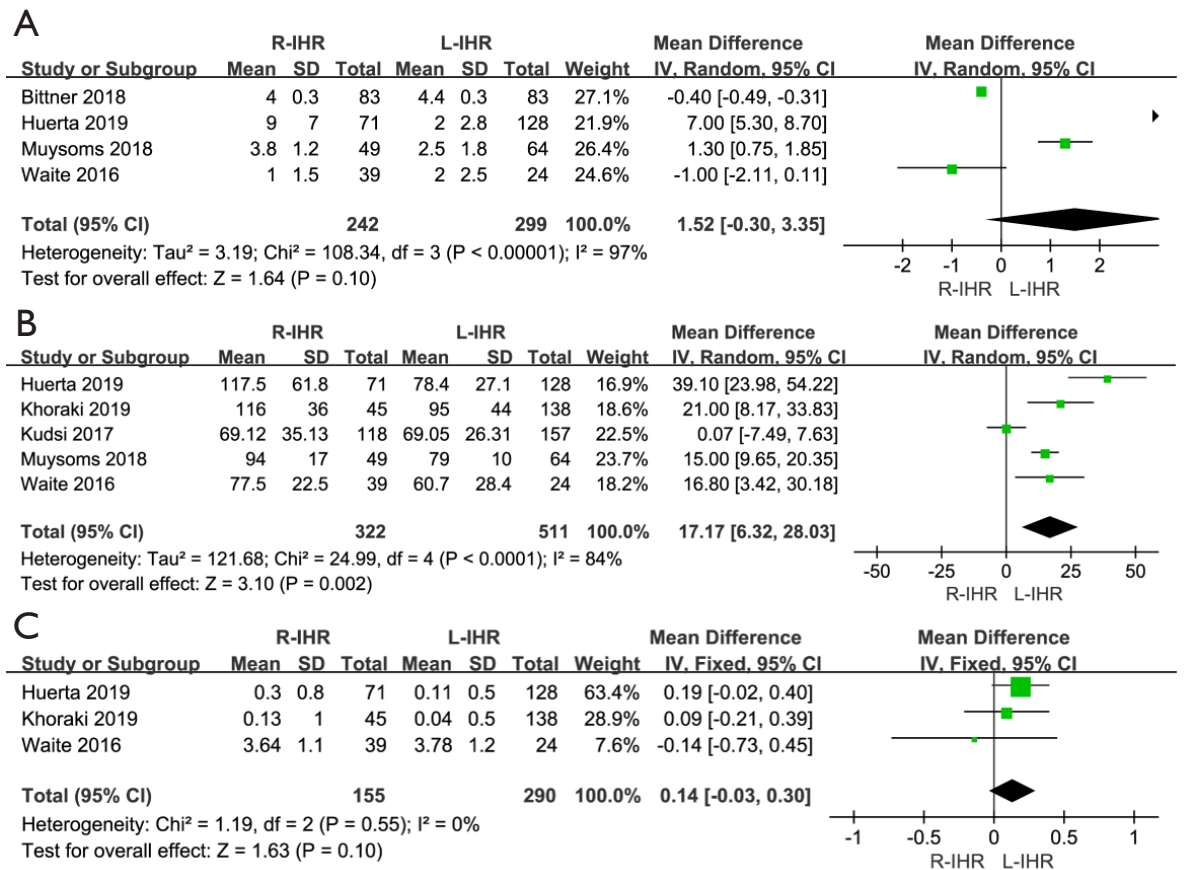

Figure 3 Meta-analysis results of pain score. (A) Forest plots of pain score in the R-IHR and L-IHR groups; (B) forest plots of operative time in the R-IHR and L-IHR groups; and (C) forest plots of length of hospital stay in the R-IHR and L-IHR groups. R-IHR, robotic inguinal hernia repair; L-IHR, laparoscopic inguinal hernia repair.

Figure $3 A$ shows a forest plot of the pain score in the R-IHR and L-IHR groups. The combined result suggested that there was no significant difference between the 2 groups $(\mathrm{MD}=1.52,95 \% \mathrm{CI},-0.30,3.35, \mathrm{P}=0.10$; $\mathrm{P}$ for heterogeneity $\left.<0.00001, \mathrm{I}^{2}=97 \%\right)$.

\section{Comparison of operative time between R-IHR and L-IHR}

Five of the included studies compared the operative time between Caucasian patients treated with R-IHR and L-IHR. Figure $3 B$ shows a forest plot of the operative time in the R-IHR and L-IHR groups. The meta-analysis results showed that the operative time differed significantly between the 2 procedures, with the time in the R-IHR group being longer than that in L-IHR group (MD $=17.17,95 \% \mathrm{CI}, 6.32,28.03, \mathrm{P}=0.002$; P for heterogeneity $\left.<0.0 .0001, \mathrm{I}^{2}=84 \%\right)$.

\section{Comparison of length of hospital stay}

Three of the included articles compared the length of hospital stay between Caucasian patients treated with R-IHR and L-IHR. The heterogeneity test results showed that the overall effect of length of stay between the R-IHR and L-IHR groups was insignificant ( $M D=0.14,95 \% \mathrm{CI}$, $-0.03,0.30, \mathrm{P}$ for overall effect $=1.63, \mathrm{P}$ for heterogeneity $=0.55$ and $\mathrm{I}^{2}=0 \%$ ) (Figure $3 C$ ).

\section{Comparison of complications}

Five studies compared the complications between Caucasian patients treated with R-IHR and L-IHR, the heterogeneity test results revealed no difference between the 2 groups ( $\mathrm{RR}=1.24$ with $95 \% \mathrm{CI}, 0.94,1.63$, $\mathrm{P}$ for overall effect $=0.13, \mathrm{I}^{2}=0 \%$ and $\mathrm{P}$ of heterogeneity $=0.57$ ) (Figure 4A).

\section{Sensitivity analysis and publication bias}

According to the results of the $1^{\text {st }}$ part of the meta-analysis, the heterogeneity for pain scores was high $\left(\mathrm{I}^{2}=97 \%\right)$. This heterogeneity may have been attributable to differences in the results of the studies. As shown in Figure $4 B$, when Huerta 2019 study (13) was excluded, the $I^{2}$ changed from $97 \%$ to $95 \%$, which evidenced the reliability of that article's results. We also performed a funnel plot analysis. The funnel plot, which is shown in Figure S2, includes 


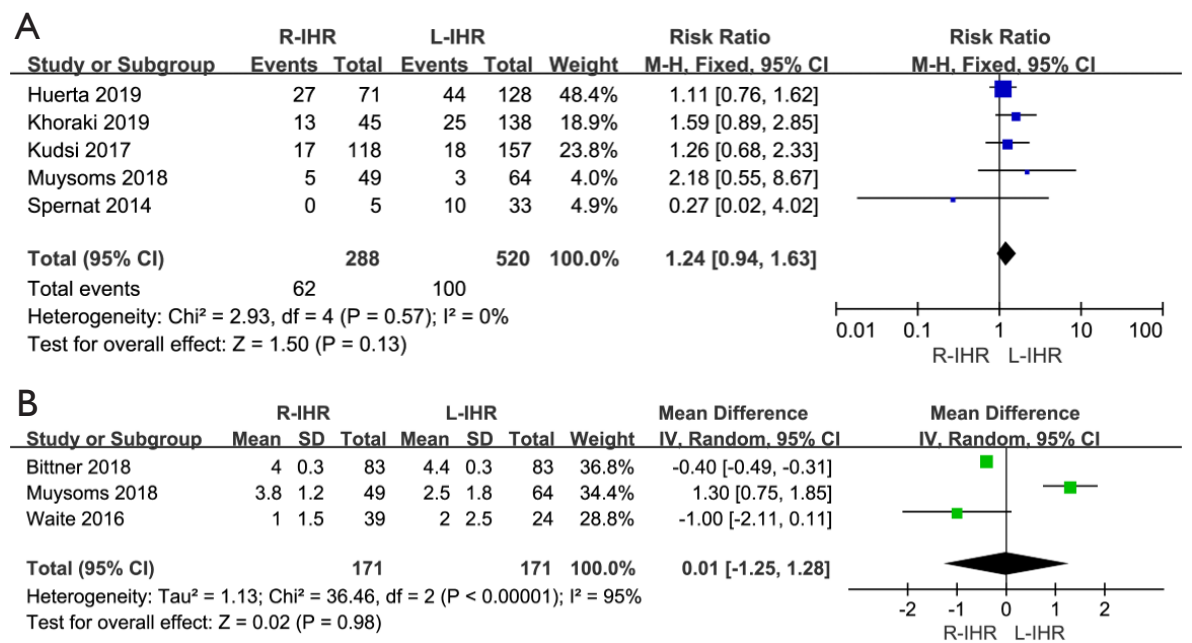

Figure 4 Meta-analysis results of complications. (A) Forest plots of complications in the R-IHR and L-IHR groups, and (B) sensitivity analysis of forest plots of pain score in the R-IHR and L-IHR groups. R-IHR, robotic inguinal hernia repair; L-IHR, laparoscopic inguinal hernia repair.

4 studies and has good symmetry, reflecting a limited publication bias.

\section{Discussion}

There are 100-300 new cases of IH per 100,000 people each year all over the world (19). Among the various types of extra-abdominal hernias, IHs are the most common. A decrease in abdominal wall muscle strength and an increase in intra-abdominal pressure are the main causes of IH $(20,21)$. The most typical symptom of an IH is a prominent, reducible mass in the groin area. At first, the mass is small, with the patient experiencing only a slight sense of bloating. However, as the mass becomes larger, pain symptoms develop. Many surgical methods for IH exist at present, and the most suitable surgical scheme should be selected according to the specific situation of each patient.

Laparoscopic surgery is performed by first puncturing the cannula under the umbilicus and puncturing the trocar. After all trocars have been implanted, they are connected to a pneumoperitoneum and observed in the abdominal cavity $(22,23)$. Patients undergoing $\mathrm{Da}$ Vinci robotic transperitoneal hernia surgery also require endotracheal intubation and general anesthesia (24).

In their study (7), Spernat et al. compared R-IHR $(\mathrm{n}=118)$ and L-IHR ( $\mathrm{n}=157)$, and showed that although the treatment received by patients in the R-IHR group was more complex, the short-term effects of the 2 procedures were comparable. At 4 weeks after surgery, there was no significant difference in the EuraHS quality of life score between the R-IHR and L-IHR groups. Our study also failed to find a significant difference between the 2 groups, which is consistent with Spernat et al.'s observations. Horovitz et al. (24) reported that there was no significant difference between the 2 groups with respect to hospital service levels. In our study of Caucasian patients, the hospital stay between the R-IHR and L-IHR groups was not significantly different. This study found that the rates of adverse events, readmission, and death were similar between the 2 groups. Furthermore, there were no differences in terms of 30-day postoperative complications after R-IHR and L-IHR, and the difference in the complications rate between the R-IHR and L-IHR groups was also insignificant.

Horovitz et al. (24) found that although the robot can provide excellent visualization and dissection of the preperitoneal space, it was associated with a longer operative time. Suture closure of the peritoneal flap appears to account for this difference. Our meta-analysis also showed that the operative time in the R-IHR group was longer than that in the L-IHR group.

One of the biggest concerns regarding robotic repair of IHs is the cost. However, most institutions with robotic systems have purchased robotic systems for different programs in many professional fields; in this respect, they are no different to laparoscopic operating towers or 
operating tables. The cost of capital is amortized over time and across all patients treated at the facility, rather than only those who receive robotic surgery. Khoraki et al. (14) reported that the average direct cost per case in the L-IHR group was $\$ 3,216$, compared with $\$ 3,479$ in the R-IHR group. The cost of surgery does not seem to be a major consideration in choosing between the 2 procedures.

Robotic technology has gradually entered the surgical thinking, and changed the surgical procedures. With the increasing application of robotic platforms in general surgery, surgeons with minimally invasive surgery experience should be familiar with robot inguinal repair technology. In addition, the selection of robotic methods may provide the selected patients with better acute pain perception and the benefits of reducing activity disruption (12).

In conclusion, considering the results of pain score, surgical complexity, and safety, R-IHR and L-IHR have similar clinical effects and safety for Caucasian patients. However, more analyses of indications for R-IHR and L-IHR in Caucasian patients should be performed in the future. Also, further research should consider additional updated articles from other countries.

\section{Acknowledgments}

Funding: None.

\section{Footnote}

Reporting Checklist: The authors have completed the PRISMA reporting checklist. Available at http://dx.doi. org/10.21037/atm-21-2126

Conflicts of Interest: All authors have completed the ICMJE uniform disclosure form (available at http://dx.doi. org/10.21037/atm-21-2126). The authors have no conflicts of interest to declare.

Ethical Statement: The authors are accountable for all aspects of the work in ensuring that questions related to the accuracy or integrity of any part of the work are appropriately investigated and resolved.

Open Access Statement: This is an Open Access article distributed in accordance with the Creative Commons Attribution-NonCommercial-NoDerivs 4.0 International License (CC BY-NC-ND 4.0), which permits the noncommercial replication and distribution of the article with the strict proviso that no changes or edits are made and the original work is properly cited (including links to both the formal publication through the relevant DOI and the license). See: https://creativecommons.org/licenses/by-nc-nd/4.0/.

\section{References}

1. Cetrulo LN, Harmon J, Ortiz J, et al. Case report of a robotic-assisted laparoscopic repair of a giant incarcerated recurrent inguinal hernia containing bladder and ureters. Int J Med Robot 2015;11:15-7.

2. Matsui S, Nitori N, Kato A, et al. Laparoscopic totally extra-peritoneal hernia repair for bilateral Spigelian hernias and coincident inguinal hernia: A case report. Int J Surg Case Rep 2016;28:169-72.

3. Feliu X, Torres G, Viñas X, et al. Preperitoneal repair for recurrent inguinal hernia: Laparoscopic and open approach. Hernia 2004;8:113-6.

4. Beets GL, Dirksen CD, Go PM, et al. Open or laparoscopic preperitoneal mesh repair for recurrent inguinal hernia? A randomized controlled trial. Surg Endosc 1999;13:323-7.

5. Dirksen CD, Beets GL, Go PM, et al. Bassini repair compared with laparoscopic repair for primary inguinal hernia: a randomised controlled trial. Eur J Surg 1998;164:439-47.

6. Sinha R, Sharma N, Dhobal D, et al. Laparoscopic total extraperitoneal repair versus anterior preperitoneal repair for inguinal hernia. Hernia 2006;10:187-91.

7. Spernat D, Sofield D, Moon D, et al. Implications of laparoscopic inguinal hernia repair on open, laparoscopic, and robotic radical prostatectomy. Prostate Int; 2014;2:8-11.

8. Arcerito M, Changchien E, Bernal O, et al. Robotic Inguinal Hernia Repair: Technique and Early Experience. Am Surg 2016;82:1014-7.

9. Yheulon CG, Maxwell DW, Balla FM, et al. Roboticassisted Laparoscopic Repair of Scrotal Inguinal Hernias. Surg Laparosc Endosc Percutan Tech 2018;28:188-92.

10. Fegade S. Laparoscopic versus Open Repair of Inguinal Hernia. World J Laparosc Surg 2008;312:309-9.

11. Higgins JPT, Altman DG, Gøtzsche PC, et al. The Cochrane Collaboration's tool for assessing risk of bias in randomised trials. BMJ 2011;343:d5928.

12. Bittner Iv JG, Cesnik LW, Kirwan T, et al. Patient perceptions of acute pain and activity disruption following inguinal hernia repair: a propensity-matched comparison of robotic-assisted, laparoscopic, and open approaches. J 
Robot Surg 2018;12:625-32.

13. Huerta S, Timmerman C, Argo M, et al. Open, Laparoscopic, and Robotic Inguinal Hernia Repair: Outcomes and Predictors of Complications. J Surg Res 2019;241:119-27.

14. Khoraki J, Gomez P, Mazzini G, et al. Perioperative outcomes and cost of robotic-assisted versus laparoscopic inguinal hernia repair. Surg Endosc 2020;34:3496-07.

15. Kudsi OY, McCarty JC, Paluvoi N, et al. Transition from Laparoscopic Totally Extraperitoneal Inguinal Hernia Repair to Robotic Transabdominal Preperitoneal Inguinal Hernia Repair: A Retrospective Review of a Single Surgeon's Experience. World J Surg 2017;41:2251-7.

16. Muysoms F, Van Cleven S, Kyle-Leinhase I, et al. Roboticassisted laparoscopic groin hernia repair: observational case- control study on the operative time during the learning curve. Surg Endosc 2018;32:4850-9.

17. Sheldon RR, Do WS, Weiss JB, et al. Sage wisdom or anecdotal dictum? Equivalent opioid use after open, laparoscopic, and robotic inguinal hernia repair. Am J Surg 2019;217:839-42.

18. Waite K, Herman M, Doyle P. Comparison of robotic

Cite this article as: Zhao F, Wang B, Chen J. Comparison between robotic and laparoscopic inguinal hernia repair in Caucasian patients: a systematic review and meta-analysis. Ann Transl Med 2021;9(10):885. doi: 10.21037/atm-21-2126 versus laparoscopic transabdominal preperitoneal (TAPP) inguinal hernia repair. J Robot Surg 2016;10:239-44.

19. Kingsnorth A. Treating inguinal hernias. BMJ 2004;328:59-60.

20. Kyle CC, Hong MKH, Challacombe BJ, et al. Outcomes after concurrent inguinal hernia repair and robotic-assisted radical prostatectomy. J Robot Surg 2010;4:217-20.

21. Iraniha A, Peloquin J. Long-term quality of life and outcomes following robotic assisted TAPP inguinal hernia repair. J Robot Surg 2018;12:261-9.

22. Hawasli A, Thao U, Chapital A. Laparoscopic Transabdominal Preperitoneal Inguinal Hernia Repair for Recurrent Inguinal Hernia. Am Surg 2002;68:303-7.

23. Kamat M, Momin ER, Upadhye AS, et al. Laparoscopic total extraperitoneal mesh repair for recurrent Inguinal Hernia: a two year experience. Indian Pract 2016;69:33-4.

24. Horovitz D, Feng C, Messing E, et al. Extraperitoneal vs. transperitoneal robot-assisted radical prostatectomy in patients with a history of prior inguinal hernia repair with mesh. J Robot Surg 2017;11:447-54.

(English Language Editor: J. Reynolds) 


\section{Supplementary}

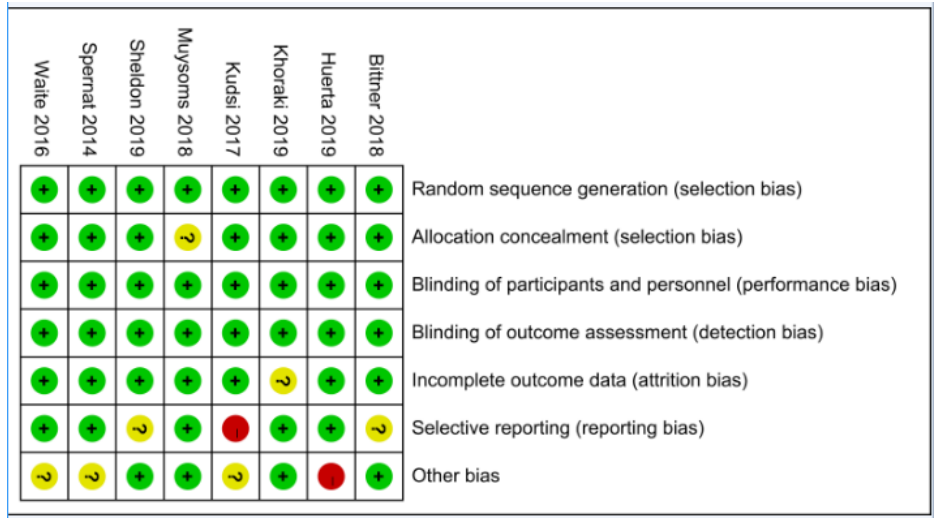

Figure S1 Quality assessment of included studies. Green represents as low risk of bias, yellow represents as unclear risk of bias, and red represents as high risk of bias.

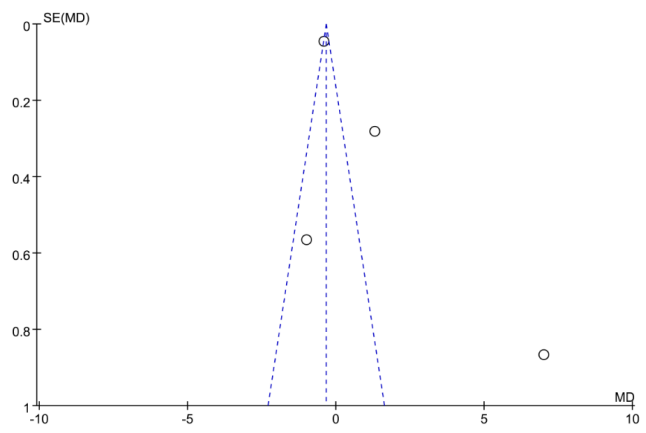

Figure S2 Funnel plot of publication bias. 